\title{
Taller Poético (1936-1938) : une crise poétique latente
}

Isabelle Pouzet

\section{(2) OpenEdition}

9 Journals

\section{Édition électronique}

URL : https://journals.openedition.org/cher/3515

DOI : $10.4000 /$ cher.3515

ISSN : 2803-5992

\section{Éditeur}

Presses universitaires de Strasbourg

\section{Édition imprimée}

Date de publication : 1 décembre 2015

Pagination : 65-72

ISBN : 978-2-86820-913-9

ISSN : 1968-035X

\section{Référence électronique}

Isabelle Pouzet, «Taller Poético (1936-1938) : une crise poétique latente », reCHERches [En ligne], 15 | 2015, mis en ligne le 01 décembre 2021, consulté le 15 décembre 2021. URL : http:// journals.openedition.org/cher/3515; DOI : https://doi.org/10.4000/cher.3515

\section{(c) (i) (2)(2)}

Ce(tte) œuvre est mise à disposition selon les termes de la Licence Creative Commons Attribution Pas d'Utilisation Commerciale - Partage dans les Mêmes Conditions 4.0 International. 


\title{
Taller Poético (1936-1938): une crise poétique latente
}

\author{
ISABELLE POUZET \\ Université Rennes 2
}

$\mathrm{L}$ es deux revues littéraires qui ont marqué la première moitié du $\mathrm{xx}^{\mathrm{e}}$ siècle

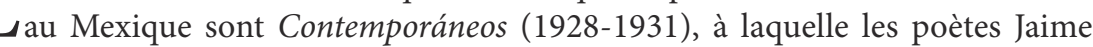
Torres Bodet, Bernardo Ortiz de Montellano, Gilberto Owen, Carlos Pellicer, José Gorostiza, Enrique González Rojo, Salvador Novo et Xavier Villaurrutia ont collaboré, et la revue Taller (1938-1941), fondée dix ans plus tard par Octavio Paz, Rafael Solana, Alberto Quintero Álvarez et Efraín Huerta. Connue pour les premiers écrits théoriques d'Octavio Paz ainsi que pour ceux des poètes et écrivains espagnols exilés après la guerre civile espagnole, la revue Taller a permis de sceller une nouvelle vision de la poésie, distincte de celle des «Contemporáneos", les collaborateurs de la revue du même nom. Si Taller a été abondamment commentée, en revanche, la revue qui l'a précédée, Taller Poético (1936-1938), fondée par Rafael Solana, a été quelque peu oubliée de la critique. Elle témoigne pourtant des hésitations et des doutes d'une génération de jeunes artistes au carrefour de plusieurs influences: d'une part, celle des mouvements d'avant-garde européens et latino-américains et, d'autre part, celle des poètes de la génération qui, au Mexique, précède ces auteurs, à savoir les "Contemporáneos». Si Taller offre à cette nouvelle génération la possibilité de se libérer de l'influence poétique des "Contemporáneos", Taller Poético témoigne au contraire d'une présence encore très forte de ces auteurs. Nous montrerons que l'apparente absence de rupture entre la nouvelle génération de poètes et les «Contemporáneos» dans cette revue est, selon nous, le signe de la difficulté de ces auteurs à se libérer du poids de leurs aînés. En d'autres termes, l'absence de crise au sein de cette revue trahit une crise nettement plus profonde qui tendrait à entraver le cheminement créateur de ces poètes.

La revue Taller Poético ne comprend que quatre numéros, parus chacun à plusieurs mois d'intervalle et de manière irrégulière. Le premier numéro sort en mai 1936, le deuxième en novembre 1936, le troisième en mars 1937 et le

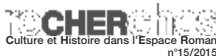


dernier plus d'un an après, en juin 1938. Son fondateur, Rafael Solana, avait commencé ses études à l'École Nationale Préparatoire, située dans l’ancien Colegio San Ildefonso à Mexico (Solana 1963: 187). Il était dans la même classe que Carmen Toscano et Efraín Huerta, deux des collaborateurs de Taller Poético. À l'EPN, il avait également fait la connaissance d'Octavio Paz, qui appartenait à la promotion antérieure. La revue Taller Poético était une revue à tirage réduit, que Rafael Solana vendait lui-même à ses camarades. Miguel Nicolás Lira, qui disposait d'une petite presse, en avait réalisé les trois premiers numéros (Solana 1963: 191).

Comme l'indique son nom, cette revue ne proposait que des textes poétiques et excluait des fragments d'œuvres théâtrales ou de textes narratifs. Peut-être cette restriction explique-t-elle le manque de régularité dans la parution des numéros ainsi que leur nombre limité. La revue a suscité bien peu d'intérêt de la part de la critique qui a préféré s’arrêter sur Taller, celle qui devait lui succéder. Pourtant Taller Poético n'est pas dénuée d'intérêt. Elle brille par sa singularité et dénote dans le contexte culturel des années 1930 au Mexique.

Comme dans toute revue de jeunes artistes, qui plus est une revue estudiantine, il n'est pas étonnant d'y trouver un véritable bouillonnement créateur: tout en proposant de nouvelles idées, elle met souvent à mal ce qui a été réalisé auparavant. C'est là une rupture naturelle qui permet de créer un espace dans lequel peut émerger la nouveauté. La revue devient alors à la fois un espace d'affirmation de soi et un espace de création. Aussi une crise, au sens de perturbation, serait-elle attendue dans ce type de publication. Or, la revue de Rafael Solana n'adopte pas une telle position puisquau lieu de rompre avec la tradition, elle sollicite les «Contemporáneos». À ce propos, trente ans après l'aventure Taller, Rafael Solana déclare:

Por contraste con todas las revistas anteriores, que habían sido agresivas, o por lo menos desdeñosas para los poetas maduros, la mía invitaba a todos los ilustres a dictarnos su lección [...] (Solana 1963: 192).

Il situe clairement le travail des jeunes collaborateurs de la revue dans le sillage de leurs prédécesseurs en présentant Taller Poético comme une revue d'union. Bien qu'il n'en fasse pas mention, l'absence de rupture avec le passé constitue, nous semble-t-il, le signe que ces jeunes auteurs ont rencontré des difficultés à s'affirmer artistiquement.

De là découle l'hypothèse sur laquelle se fonde notre travail, qui peut être exprimée de la façon suivante: l'absence de crise apparente - au sens de rupture - pourrait en réalité cacher une crise beaucoup plus profonde affectant les jeunes collaborateurs de cette revue. Elle traduirait leur difficulté à se détacher de la tradition et en particulier de la poésie des «Contemporáneos».

Taller Poético se distingue des revues qui l’ont précédée et même de celles que certains de ses collaborateurs ont fondées. Nous pensons à Barandal d'Octavio Paz, de Salvador Toscano et d'Arnulfo Martínez Lavalle créée en 1931, dont la publication s'est arrêtée en 1932; ou encore à Cuadernos del Valle de México 
qui lui a succédé (1933-1934). Ces deux revues, à la différence de Taller Poético, nétaient pas consacrées uniquement à la poésie. La première est parue alors que ses fondateurs, à peine âgés de dix-sept ans, étaient étudiants à l'EPN. Barandal s'inscrivait certes dans le sillage de la célèbre revue Contemporáneos (1928-1931) qui avait marqué, au Mexique, les années 1930, mais cherchait malgré tout à se démarquer de l'influence des aînés. Dans la première section, ses collaborateurs proposaient un article critique, un poème ou bien un extrait de pièce de théâtre dont ils étaient eux-mêmes les auteurs. La deuxième section de la revue incluait l'article d'un auteur reconnu portant sur un thème culturel. La troisième partie était consacrée aux nouveautés littéraires. À partir du troisième numéro, Barandal proposait un supplément de poésie dans lequel figuraient les textes de certains des "Contemporáneos». Trois poèmes inédits de Carlos Pellicer apparaissent ainsi dans le troisième numéro, des passages de Lota de loco de Salvador Novo composent le supplément du quatrième numéro, et les poèmes «Nocturno» et "Nocturno eterno» de Xavier Villaurrutia sont inclus dans le numéro de décembre 1931. La présence des «Contemporáneos» dans Barandal s'explique par l'admiration que ses collaborateurs leur portaient, par la facilité avec laquelle ils avaient pu entrer en contact avec eux - rappelons que Carlos Pellicer avait été professeur de littérature à l'EPN - et surtout par le prestige que leurs noms conféraient à cette publication.

Dans Cuadernos del Valle de México, cette présence disparaît. Ses fondateurs ne font allusion ni aux "Contemporáneos» ni même à leurs œuvres. La revue devient un véritable espace de création pour ces jeunes auteurs qui semblent sêtre enfin libérés du poids de leurs aînés. Elle est par ailleurs fortement imprégnée de leur intérêt pour le communisme. Dans les deux seuls numéros parus, plusieurs articles en témoignent: les deux nouveaux collaborateurs de la revue, Enrique Ramírez y Ramírez et José Alvarado ont consacré plusieurs articles à la révolution russe.

Il semblait donc logique de s'attendre à ce qu'une revue qui rassemble les fondateurs de Barandal et de Cuadernos del Valle de México, c'est-à-dire Octavio Paz, Arnulfo Martínez Lavalle, Salvador Toscano, Rafael López Malo, José Alvarado et Enrique Ramírez y Ramírez, ignore la poésie des «Contemporáneos ». Rafael Solana aurait pu chercher à imiter ces deux publications et s'engouffrer dans la voie politique tout en laissant une place conséquente à la création littéraire. Or c'est le contraire qui se produit. Non seulement il décide d'élaborer une publication entièrement consacrée à la poésie - elle ne présente pas d'articles politiques comme les deux précédentes - mais il fait intervenir à de nombreuses reprises les auteurs de la revue Contemporáneos. Des années plus tard, Rafael Solana revient sur cette caractéristique et les objectifs de la revue:

[...] lograr la concordia entre todos los poetas existentes en México; era una revista de unificación [...] la revista habría debido llamarse, para que su nombre correspondiera exactamente a su idea, Panteón, pues se trataba de levantar un templo en el que fueran adorados todos los dioses, los más antiguos y los más nuevos. (Solana 1963: 191) 
Venant d'un groupe de jeunes artistes, l'idée de créer une revue qui laisserait une place à tous les poètes mexicains surprend, d'autant plus que cela va à l'encontre de ce qui était en germe dans les deux revues culturelles qui l'ont précédée. Pourtant c'est bien l'idée qui est à l'œuvre dans Taller. La revue a toujours eu à cœur de ne pas rejeter les "Contemporáneos", comme l'a précisé Octavio Paz en 1976 dans une lettre adressée à José Emilio Pacheco:

Tampoco "denostamos" a los poetas y escritores de Contemporáneos ni ellos fueron reivindicados por nuestros amigos de Tierra Nueva. No hubo enemistad entre Tierra Nueva y Taller ni entre Taller y Contemporáneos. Salvo Efraín Huerta, que los atacó varias veces -nunca en Taller sino en El Nacional-, nosotros fuimos amigos de los "Contemporáneos". (Paz 1982: 16-17)

Contrairement à Barandal qui faisait intervenir les "Contemporáneos» en dehors de la revue, c'est-à-dire dans ses suppléments, Taller Poético intègre pleinement ces auteurs à chacun de ses numéros. Dans le premier numéro, de mai 1936, à côté des poèmes des jeunes poètes tels que Efraín Huerta, Vicente Magdaleno ou Rafael Solana, se trouvent les textes de Carlos Pellicer et de Salvador Novo ; dans le deuxième numéro, de novembre, Enrique González Rojo, Bernardo Ortiz de Montellano et Jaime Torres Bodet sont à l'honneur; dans le troisième numéro, publié en mars 1937, c'est Xavier Villaurrutia; et, enfin, les textes inédits de Jorge Cuesta et de Carlos Pellicer figurent dans le dernier numéro. La plupart des textes proposés par ces auteurs, tout comme ceux de la nouvelle génération, n’avaient encore jamais été publiés. Par exemple, le poème de Jaime Torres Bodet, intitulé «Sitio» et inclus dans le deuxième numéro (novembre 1936), ne sera publié qu'en 1937 dans son recueil Cripta (Torres Bodet 1994: 43-44). Dans le numéro suivant, de mars 1937, Xavier Villaurrutia propose lui aussi un poème, "North Carolina Blues », qui ne paraîtra que l'année suivante dans son recueil Nostalgia de la muerte (Villaurrutia 2006: 64-65). Tant pour les poètes de la jeune génération que pour leurs aînés, Taller Poético se présente comme un espace de diffusion de leurs écrits.

Néanmoins, il nous paraît surprenant d'associer des poètes dont les renommées ne sont plus à faire et de jeunes auteurs qui n'en sont qu’à leur première publication et qui, par conséquent, sont en plein "apprentissage» de l'écriture poétique. Rappelons qu'Octavio Paz a publié son premier recueil Luna silvestre en 1933, Rafael Solana Ladera l'année suivante et Efraín Huerta Absoluto amor en 1935.

Comment expliquer la présence des «Contemporáneos» dans Taller Poético? Il faut dire qu'ils ne disposent plus de revue propre depuis plusieurs années. Les revues se sont succédé et ont duré très peu de temps: Resumen, fondée en 1931 par Salvador Novo, a eu une existence d'à peine un an; Examen de Jorge Cuesta, parue en 1932, est censurée dès le troisième numéro. Par ailleurs, en mars 1932, le débat autour du nationalisme en littérature a définitivement divisé le groupe des "Contemporáneos »1. Dans ce contexte, la revue Taller Poético apparaît pour eux comme un espace idéal de diffusion de leurs textes poétiques.

1 Voir Sheridan 1999: 59. En 1932, la polémique autour du sens de la littérature et de son rapport 
Depuis 1934, Lázaro Cárdenas est au pouvoir et il est favorable à l’ouverture artistique de son pays, ce n'est donc pas le contexte politique qui a conduit Rafael Solana à faire appel à ces poètes. La raison n'en est pas davantage la renommée des «Contemporáneos» qui, par ailleurs, depuis la polémique de 1932 ont perdu quelque peu de leur prestige. C'est bien l'admiration qu'ils éveillent chez ces jeunes auteurs, et en particulier chez Rafael Solana, qui semble expliquer leur présence si forte au sein de cette revue. Toutefois, cette présence, même si elle est clairement revendiquée par Rafael Solana, pourrait constituer une entrave au développement de leur propre écriture.

Afin de confirmer cette hypothèse, il convient d'examiner le contenu des écrits que ces jeunes auteurs ont proposés dans cette revue. En considérant l'ensemble de leurs poèmes publiés dans les quatre numéros de Taller Poético, force est de constater que la poésie des «Contemporáneos» exerce une influence notable sur la jeune génération. Leurs poèmes, dont la forme n’est généralement pas figée, offrent souvent un dialogue intime entre la première personne et un interlocuteur et leur toile de fond est la relation amoureuse. La façon dont certains «Contemporáneos» ont pratiqué le dialogue intime et fui les ornements du modernisme transparaît dans les poèmes de la génération suivante. Il est clair que les poèmes de la jeune génération font écho à ceux des "Contemporáneos ". Il se dégage ainsi des textes de Taller Poético une certaine harmonie tant du point de vue isotopique que formel. Néanmoins, ces jeunes poètes ne sont pas dans l'imitation stérile de leurs aînés. Voici à titre d'exemple deux fragments de poèmes parus dans le quatrième numéro de la revue (juin 1938). Les vers reproduits ci-dessous à gauche sont de Jorge Cuesta, ceux de droite d'Efraín Huerta:

Tu voz es un eco, no te pertenece, no se extingue con el soplo que la exhala. Tus pasos se desprenden de ti y hacen caminar un fantasma intangible y [perpetuo que te expulsa del sitio donde vives tan pasajeramente te suplanta. Tanto mi tacto extremas y prolongas que al fin no toco en ti sino humo, sombras, [sueños, nada.

(Cuesta 1981: 187-188)²
Sola mi voz, caída quedamente en el pantano donde cabe ausente mi recuerdo sin rosas ni claveles. Mi voz en la saliva del olvido, como pez en agua de naufragio. [...] No lloraría por mi ternura finalmente enterrada ni por un sueño herido sentiría fina tristeza, pero si por mi voz oculta para siempre, mi voz como perla abandonada.

(Huerta 1981 : 198-199)

à la nationalité secoue le monde littéraire mexicain. La question posée par Alejandro Núñez Alonso dans El Universal Ilustrado en mars 1932 va scinder en deux groupes le microcosme littéraire de Mexico. Il y aura, d'un côté, les avant-gardistes, et, de l'autre, les nationalistes. La question "Existe-t-il une crise dans la génération d'avant-garde? » va même diviser les «Contemporáneos» puisque Salvador Novo et Ortiz de Montellano répondent qu'il n’y a pas de crise et qu'ils ont même comme disciples les poètes de Barandal alors que José Gorostiza et Samuel Ramos se rétractent et se montrent subitement favorables à une littérature plus «nationale».

2 Pour les deux citations, nous faisons référence ici au fac-similé de la revue Taller Poético publiée 
À la proximité physique de ces vers qui se trouvent à quelques pages d'intervalle dans la revue, s'ajoute une proximité isotopique: chaque auteur a construit son texte autour du motif de la «voix». Dans le contexte amoureux du premier poème, la femme aimée est désignée par ce terme. En revanche, le second fait allusion à la situation de solitude du «je» poétique, sans doute causée par la perte de l'être aimé. Les vers polymétriques, en particulier ceux de Jorge Cuesta, ne sont pas sans rappeler ceux d'un autre "Contemporáneo », Xavier Villarrutia. Ce dernier, dans «Poesía», publié en 1926 dans son recueil Reflejos, affirme: «Tu voz, hoz de eco, / es el rebote de mi voz en el muro [...]» (Villaurrutia, 2006: 26). Il ressort de la mise en regard de ces vers que les poèmes des deux auteurs se font écho sans pour autant que nous puissions parler d'imitation. En effet, il ne s'agit pas d'influence entre ces deux publications concomitantes, mais plutôt d'une "parenté» entre la jeune génération et l'ancienne. Toutes deux s'intéressent à des motifs poétiques similaires. Les jeunes auteurs développent un univers poétique propre, mais en même temps il s'installe une certaine continuité entre les deux générations, non exempte, comme nous l'avons dit, d'une certaine difficulté des plus jeunes à se détacher de leurs aînés.

Par ailleurs, les poèmes de ces jeunes auteurs laissent entrevoir leur lecture assidue de la littérature issue des mouvements d'avant-garde européens et latino-américains. Rappelons que la revue Contemporáneos avait joué un rôle fondamental dans la diffusion des nouveautés littéraires venues d'ailleurs, par exemple des poètes espagnols de la Génération de 1927, du mouvement créationniste de Vicente Huidobro ou encore des surréalistes français. Le poème de Vicente Magdaleno, publié dans le dernier numéro, fait intervenir des images de toute évidence marquées par le futurisme ou du moins par son «relais» mexicain, le stridentisme de Manuel Maples Arce:

$$
\begin{aligned}
& \text { Su mismo cielo era un cielo lúgubre... Y por } \\
& \text { las tardes } \\
& \text { flotaba sobre la llanura un polvo pardo, y las } \\
& \text { grandes torres } \\
& \text { de la energía y los anuncios luminosos, igual } \\
& \text { que la nerviosidad horrible de sus habitantes } \\
& \text { eran como los signos de una catástrofe. [...] }
\end{aligned}
$$

(Magdaleno 1981: 207-208)

L'influence de la Génération de 1927 est tout aussi perceptible dans Luna silvestre, le premier recueil d'Octavio Paz publié en 1933, et dans Absoluto Amor d'Efraín Huerta. Chacun, à sa manière, révèle l'empreinte de la lecture de l’œuvre de Juan Ramón Jiménez, notamment de La soledad sonora (1911), par le traitement du motif de la lune (Stanton 2001: 50-59).

Finalement, c'est peut-être dans la section des comptes rendus que les collaborateurs de la revue expriment un certain détachement par rapport aux «Contemporáneos». Il s’agit d’un espace de liberté où Rafael Solana, Carmen

au Mexique en 1981 par le Fondo de Cultura Económica. 
Toscano et Efraín Huerta ont pour habitude de rédiger les critiques de recueils qui viennent de paraître, en particulier ceux des collaborateurs de la revue. Or, aucun des "Contemporáneos» n’apparaît dans cette section puisqu'ils ne sont sollicités que pour proposer leurs poèmes. Aussi, cet espace «d'auto-promotion » constitue-t-il un lieu de liberté d'expression. C'est en ce sens que va l'entendre Efraín Huerta lorsqu’il rédige le compte rendu du poème "¡No pasarán!» qu'Octavio Paz a écrit en soutien aux républicains espagnols et qu'il a publié en 1937. Ce poème a secoué le monde littéraire de l'époque et a reçu de vives critiques de la part de Bernardo Ortiz de Montellano, l'un des fondateurs de Contemporáneos (Stanton 2001: 67). Efraín Huerta s'est empressé de défendre son ami par ces mots:

Paz, poeta serio y consciente, como ningún otro, ha dado a la poesía mexicana el primer documento valioso y digno; ha puesto en las manos de los críticos suspicaces algo que les quema las manos; ha entregado al pueblo de México y al de España el medio más efectivo de comunión y entendimiento. Ha creado una auténtica poesía de ilimitadas perspectivas. (Huerta 1981: 155)

Force est de le constater, une faille s'est creusée entre les «Contempo-ráneos » et cette jeune génération qui va progressivement revendiquer son besoin de créer un lien entre la poésie et la vie, lien que les «Contemporáneos» avaient volontairement effacé.

Selon Rafael Solana, la revue Taller Poético a disparu parce qu'elle avait les plus grandes difficultés à financer sa publication. De concert avec Octavio Paz, Alberto Quintero Álvarez, Efraín Huerta et Neftalí Beltrán, il décide alors de fonder une nouvelle revue qui, cette fois, n'est pas consacrée uniquement à la poésie mais s'ouvre aussi à d'autres formes d'expression culturelle. Taller est née. (Solana 1963: 196). Rafael Solana y laisse une place de choix aux "Contemporáneos» au détriment des poètes de sa propre génération.

De fait, la crise de la poésie est palpable dans Taller Poético. Octavio Paz, dans son article «Razón de ser», publié dans le deuxième numéro d’avril 1939, propose une véritable déclaration de principe de la revue. Il y explique sa vision de la poésie qui est celle quépousent également les poètes de sa génération. Il ne cherche pas l'originalité mais l'authenticité, la poésie constitue une activité vitale et non un moyen d'expression et son objectif est de changer l'homme et la société. Il fait brièvement allusion aux «Contemporáneos» pour remettre en question leur conception de la poésie et, plus encore, la dépasser:

$\mathrm{Y}$ en esta aspiración nos acompañan los que saben que la juventud no vale nada cuando deja de ser una posibilidad, un acitate y un tránsito. Tal es el sentido de Taller, que no quiere ser el sitio en donde se asfixia una generación, sino el lugar en donde se construye el mexicano, y se le rescata de la injusticia, la incultura, la frivolidad y la muerte. (Paz 1982: 154)

Taller Poético manifeste de toute évidence une crise latente qui témoigne de la difficulté pour cette nouvelle génération de poètes de se libérer de l'influence des "Contemporáneos». Créer une nouvelle revue qui laisse une place importante 
aux créations de la jeune génération et aux productions des exilés espagnols, c'est prendre ses distances par rapport à ces aînés dont l'influence et la présence étaient trop prégnantes.

\section{Bibliographie}

Cuesta, J., 1981, "Una palabra obscura», Revistas literarias mexicanas modernas. Taller Poético (1936-1938). Poesía (1938), edición facsímil, México, Fondo de Cultura Económica, p. 184-188.

Huerta, E., 1981, "Tres cantos de abandono», Revistas literarias mexicanas modernas. Taller Poético (1936-1938). Poesía (1938), edición facsímil, México, Fondo de Cultura Económica, p. 195-205.

Huerta, E., 1981, «No pasarán», Revistas literarias mexicanas modernas. Taller Poético (1936-1938). Poesía (1938), edición facsímil, México, Fondo de Cultura Económica, p. 155.

Magdaleno, V., 1981, "Atardecer sin lirios», Revistas literarias mexicanas modernas. Taller Poético (1936-1938). Poesía (1938), edición facsímil, México, Fondo de Cultura Económica, p. 207-210.

Paz, O., 1982, "Carta de Octavio Paz a José Emilio Pacheco», Revistas literarias mexicanas modernas. Taller I-VI. Diciembre de 1938-Noviembre de 1939, edición facsímil, vol. I, México, Fondo de Cultura Económica, p. 16-17.

Paz, O., 1982, "Razón de ser», Revistas literarias mexicanas modernas. Taller I-VI. Diciembre de 1938-Noviembre de 1939, edición facsímil, vol. I, México, Fondo de Cultura Económica, p. 150-154.

Sheridan, G., 1999, México en 1932: la polémica nacionalista, México, Fondo de Cultura Económica.

Solana, R., 1963, «Barandal, Taller Poético, Taller, Tierra Nueva», Las revistas literarias de México, México, Instituto Nacional de Bellas Artes, p. 185-207.

Stanton, A., 2001, Las primeras voces del poeta Octavio Paz (1931-1938), México, Conaculta.

Torres Bodet, J., 1994, Obras escogidas. Poesía. Autobiografía. Ensayo, México, Fondo de Cultura Económica.

Villaurrutia, X., 2006, Obras: poesía, teatro, prosas varias, crítica, México, Fondo de Cultura Económica. 


\title{
Crise économique et misère sociale dans Roble huacho (1947) de Daniel Belmar (Chili)
}

\author{
Benoît SANTINI \\ Université du Littoral Côte d'Opale (Boulogne-sur-Mer) \\ Laboratoire HLLI
}

\begin{abstract}
A près une période de prospérité et de stabilité économique entre 1926 et 1929, le Chili subit de plein fouet la crise économique mondiale entre 1929 et 1932. Comme l'explique Luis Riveros, «la depresión económica vivida por Chile se derivó de la magnitud del efecto que la crisis internacional indujo en la balanza de pagos», et l'auteur souligne "el derrumbe de la economía chilena» à partir de 1931 (Riveros 2009: 1, 4). Roberto Toso et Álvaro Feller précisent: «El peor momento de la crisis se vivió durante 1932» (Toso-Feller 1983: 15). Le secteur minier, lié à l'exploitation du salpêtre, produit fortement touché depuis la fin de la Première Guerre mondiale, fut d'autant plus affecté par la crise, comme l’expose Sergio Villalobos: «la desocupación en la región salitrera alcanzó proporciones catastróficas y grandes grupos de obreros cesantes debieron ser trasladados al centro del país» (Villalobos 1983: 193). On assista à une chute de la production du cuivre, à une baisse de $33 \%$ des exportations et de $60 \%$ des importations, quant au PIB en 1937, il ne retrouva pas son niveau d'avantcrise (Sosnowski 1997: 293). Villalobos rappelle: "fue necesario que el Estado proporcionase alimentación diaria a la masa de desocupados», "tan grande fue la crisis que sus efectos se dejaron sentir por muchos años" (Villalobos 1983: 193). Ernesto Bohoslavsky, pour sa part, analyse la situation en ces termes:

En 1932 [...] Chile era el país más afectado en el mundo por la Depresión desatada dos años atrás. La caída en los precios de los productos primarios afectó gravemente a las exportaciones nacionales. [...] Aunque la crisis salitrera se venía arrastrando desde mediados de la década de 1920, estos años no hicieron sino profundizar el conflicto, arrojando a varios miles de mineros desempleados a los centros urbanos del país. En 1931 se agravó aun más la situación ya que cerraron más empresas dedicadas al cobre, al salitre y al carbón. [...] La Araucanía, región eminentemente agrícola, acusó recibo del galopante desempleo. Por su
\end{abstract}

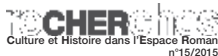


configuración minifundista de la tierra, Cautín era una de las provincias en las que había una proporción mayor de pequeñas propiedades agrarias familiares. La acentuada presencia de explotaciones familiares generó una rigidez del mercado de trabajo rural que dificultaba la potencial absorción de los parados (Bohoslavsky 2002: 547).

Ainsi, le Chili vit-il des années noires, et se retrouve-t-il confronté à une situation dramatique de misère et de chômage.

À cette époque (années 1920-1930), la création littéraire au Chili connaît un bel essor et la préoccupation sociale y occupe une large place. En poésie, Pablo Neruda, Gabriela Mistral ou Pablo de Rokha publient des œuvres essentielles de leur production ${ }^{1}$. Le dramaturge Antonio Acevedo Hernández publie en 1936 la pièce Chañarcillo, dont l'action se situe au cours de la première moitié du XIx ${ }^{e}$ siècle, en plein essor de l'exploitation du cuivre. En 1933 parait Llampo brujo de Sady Zañartu, roman qui aborde la question minière (Silva Castro 1960 : 65). C’est la "Generación de 1938 » plus particulièrement qui s’intéresse à la problématique sociale, comme en attestent les romans de Nicomedes Guzmán, Andrés Sabella ou encore Daniel Belmar. Cependant, peu de romans, pièces de théâtre ou poèmes se centrent sur la crise économique de l'époque en tant que telle, à la différence de la riche production nord-américaine traitant du sujet $^{2}$. Il faudra attendre 1947 pour que Daniel Belmar publie son roman Roble Huacho où il déploie diverses stratégies narratives afin d'évoquer les effets de la Grande Dépression au Chili entre 1929 et 1933. Peu de dates y sont précisées, mais le roman invite le lecteur à déployer toute son imagination et sa capacité à mettre en relation, à associer l'histoire ou les histoires narrées au contexte socioéconomique de ces années. Daniel Belmar (1906-1991), lauréat de nombreux

1 Rappelons l'engagement de P. Neruda, l'appartenance au communisme de P. de Rokha, la composition de poèmes sociaux par G. Mistral. Pablo Neruda publie en effet Residencia en la tierra en 1927 et España en el corazón en 1937, Gabriela Mistral Tala en 1938, Pablo de Rokha Canto de trinchera 1929-1933. N'oublions pas le recueil avant-gardiste de Vicente Huidobro, Altazor (1931), preuve de la vitalité créatrice dans le Chili de l'époque.

2 Consulter le document suivant donnant des précisions sur la littérature nordaméricaine et la crise de 1929: http://www.bpi.fr/modules/resources/download/ default/Decouvrir_la_Bpi/Documents/Bibliographies/ecrivains29.pdf

Comme l'écrit María Orozco Jesús Vera, «Entre los años 1935 y 1950 comenzaron a publicar novelistas que componen la generación de 1942, Generación Neorrealista o Generación de 1938, según las distintas denominaciones de la crítica. Jacobo Danke, Daniel Belmar, Nicasio Tangol, Gonzalo Drago, Coloane, Mariano Bahamonde, Oscar Castro, Reinado Lomboy, $\mathrm{M}^{\mathrm{a}}$ Carolina Geel y Fernando Alegría, entre otros, componen este grupo literario en el que se incluye también $\mathrm{M}^{\mathrm{a}}$ Luisa Bombal. Frente a la literatura criollista estos escritores proponen una estética que supere las limitaciones regionalistas y que contemple al hombre desde otra óptica. Su narrativa, inmersa en un profundo lirismo, se concreta en la búsqueda de la identidad del hombre, ese incesante bucear en las profundidades de la mente humana que le ofrecen las nuevas corrientes vanguardistas, especialmente el surrealismo" (Orozco Jesús Vera 1989: 39-40). 
prix littéraires, est chimiste et pharmacien de formation. La toile de fond de ses romans est très souvent la région de Concepción (VIIIe région, au nord de l'Araucanie). Roble huacho est le roman qui le fait connaître. Dans Coirón (1949), il se remémore son enfance à Neuquén, en Argentine. En 1955, il publie Los túneles morados, dont l'histoire se déroule à Concepción. Detrás de las máscaras, qui paraît en 1965, est écrit peu de temps après les cataclysmes des 21 et 22 mai 1960 qui détruisirent les villes du sud chilien et de Chiloé. Ainsi, ancre-t-il son écriture dans les régions du Chili qui deviennent le décor essentiel de ses romans.

Roble huacho, composé de seize chapitres, relate la misère d'un village du même nom situé dans la région de Cautín (IX ${ }^{\mathrm{e}}$ région, Araucanie). Son pharmacien Pancho Ríos, également narrateur homodiégétique en focalisation interne, brosse une série de portraits de villageois sans-le-sou, mais rapporte aussi des épisodes où il partage des moments complices avec ses amis et sa compagne, Solveig, rencontrée au cours du roman, une jeune fille mal mariée qui devra rejoindre de force son époux vivant à Puerto Saavedra (en Araucanie). Le roman nous amène à nous poser la question suivante: dans quelle mesure l'écriture narrative de Belmar dans Roble huacho ainsi que ses personnages sontils affectés par la dépression économique et comment ce roman parvient-il à «éclairer le concept de crise», pour reprendre les propos d'Edgar Morin (Morin 1976: 149)?

\section{Reflet de la crise économique dans Roble huacho}

La crise économique est le facteur principal de la déliquescence du village de Roble huacho, "poblacho» ou "pequeño pueblo de la antigua frontera mapuche» (Belmar 1947: 24), et le signifiant-clé du roman. Tout au long de l'œuvre, la récurrence lexicale du terme "crisis» ponctue et envahit l'énoncé, le dévastant: "¿Cuándo pasará esta maldita crisis?» (Belmar 1947: 18), se demande le narrateur, pharmacien de son état; il est fait allusion à «la más intensa de las crisis financieras que soportara el país» (Belmar 1947: 24) et le narrateur explique: «Hace ya mucho, antes que la crisis asomara su careta trágica sobre los campos ubérrimos, cada madrugada volcaba sobre el pueblo un desfile interminable de carretas fleteras», ou encore: «Es la crisis, y su angustia irremediable golpeando para nada» (Belmar 1947: 35, 37). Ce même narrateur ajoute, à propos de «El Guata $»^{3}$ Bascur, ferronnier: "Ahora, con la crisis se ha desbandado toda su clientela", "Hoy, la crisis ha transformado estas fondas en cantinas y prostíbulos clandestinos», "Los campesinos, atemorizados por la crisis, apenas sembraron este año lo indispensable para subsistir» (Belmar 1947 : 71, 86, 144). Il précise encore : «La crisis abruma el poblacho», «La crisis ha puesto en evidencia la fragilidad financiera de ciertos hacendados» (Belmar

3 En mapudungün, langue des Mapuches, «huata» signifie "estómago». «El Guata» Bascur «debe el sobrenombre al descomunal desarrollo de su panza» (Belmar 1947: 70). 
1947: 172) et évoque le destin d'un: «jornalero agrícola que, acorralado por la crisis en las secas lomas de Nuble 4 , bajara hacia el sur» (Belmar 1947: 198). Belmar multiplie les termes appartenant à la constellation sémantique de la crise: «fragilidad financiera», «deudas», «quiebras», «depresión» (Belmar 1947: 172-173). Ces allusions à la crise envahissent l'espace textuel comme la crise déferle sur le monde en 1929-1933. De façon insidieuse, celle-ci s'immisce progressivement dans le quotidien des habitants du village et dans certains secteurs-clé (secteur agricole), elle s'infiltre au fil des pages du roman et se propage telle une épidémie, confirmant ce quécrit Jean Baudrillard qui considère la crise comme une "catastrophe au ralenti» (Baudrillard 1990: 41).

La dépression économique crée un manque et un vide au sein de la population de Roble huacho, reproduction en miniature du Chili de l'époque. Aussi, soulignons-nous la portée signifiante des négations qui mettent en exergue ces carences: "Hace ya dos años que no me compro ni una camisa. [...] La gente no tiene ni para comer", "nadie compra nada. No hay dinero", «tampoco tengo nada. Ni siquiera un pollito que vender» (Belmar 1947: 18, 37, 42). Les négations deviennent l'un des traits distinctifs de l'écriture de Belmar. S’y associent en outre des termes évoquant l'absence: "privaciones, mínima cuantía, escasez de movimiento", "qué privaciones voy a tener que sufrir», «calle solitaria», «magros negocios» (Belmar 1947: 25, 28, 36). Lénonciation est ainsi rongée par les effets de la crise. Graphiquement, cette absence se manifeste aussi par les très nombreux points de suspension présents dans les conversations. Par exemple, lors d'une discussion entre Tito Andrade, petit employé de voirie, et «el rucio» Fernández, directeur de la succursale de la Caisse d'Epargne du village, ce dernier s’écrie: «No seas 'roto'... ¿de qué te quejas?... Tienes un buen sueldo... viáticos... y nadie a quien ayudar... En cambio yo... mi madre todo el tiempo enferma... y todavía estoy devolviendo el dinero que entregué a ese maricón desgraciado» (Belmar 1947: 27). Fernández fait référence au directeur de la Caisse d'Épargne de Pichi-Lleuque qui aurait malhonnêtement conservé une partie de la somme des «cheques fiscales» de la commune, remis par Tito, obligeant ce dernier à rembourser la différence. Les points de suspension abondent encore lorsque, après un flash-back narratif, alors que le narrateur lui conseille de s'adresser à Don Casimiro pour qu'il lui prête de l'argent, Tito lui répond: «Psss... Cien pesos a lo más... ¿sabes cuánto gano?... Apenas trescientos noventa pesos, con los descuentos... ¡Maricón desgraciado!... quizás qué privaciones voy a tener que sufrir por su culpa!...» (Belmar 1947: 27). Ces silences narratifs et vides verbaux confirment que «la littérature se définit [...] comme une herméneutique du silence» (Sartre 1972:29) et que "le silence est un moment du langage» (Sartre 1948: 32), «un acte verbal, un trou creusé dans le langage» (Louette 1995: 36). Si la crise ruine l'individu et mine son niveau de vie, elle semble creuser ses sillons dans le langage de certains personnages du roman, durement touchés par la précarité. Tito Andrade en vient d'ailleurs

4 Nuble se situe dans la région du Bío Bío, au nord de l'Araucanie. 
à sécrier, dans le chapitre I: «¿Qué año apocalíptico!»(Belmar 1947: 29). Nous avons affaire à une réécriture de l'Apocalypse biblique où s'active la «relation de coprésence» "entre deux textes», décrite par Gérard Genette (1982: 8), ici Le Nouveau Testament et Roble huacho, au service d'une dénonciation. Si les méfaits de Lucifer laissent la place au millénium, le village de Roble huacho, touché par la crise, la maladie et autres calamités, semble avoir atteint la fin d'une étape menant vers une re-naissance.

Face aux pauvres gens, apparaît une oligarchie opulente incarnée par Don Marcelo ( agente maderero») et Don Andrés ( «comerciante»). Ces derniers sont définis en ces termes: «Son poderosos». Don Nacho Cabrera, grand propriétaire, est désigné par son «estatura feudal» et «sus vastos dominios» (Belmar 1947: 50). L'aisance matérielle de Don Segundo est soulignée avec insistance: "Don Segundo es rico. Tiene fortuna" (Belmar 1947: 53). Ces insistances créent un contraste avec les carences des villageois de Roble huacho. Daniel Belmar, par l'implicite et l'habileté langagière, dénonce le pouvoir des groupes économiques et sociaux dominants si peu affectés par la crise.

Les habitants du village sont victimes de la misère et du chômage. Comme nous l'avons dit en introduction, le secteur minier - en particulier, l'exploitation du salpêtre - est particulièrement touché par la Grande Dépression. Ainsi, le narrateur fait-il allusion aux «cesantes de las salitreras»(Belmar 1947: 37), reconvertis en cantonniers à cause de la crise. Il évoque les déplacements et les mutations provoqués par celle-ci: «La semana pasada se fue Tito Andrade, trasladado al Departamento de Caminos de la provincia de Malloco», la succursale de la Caisse d'Épargne ayant en effet fermé "por escasez de movimiento» (Belmar 1947: 194). Est également mentionnée la précarité des logements d'une frange de la population qui vit dans des bidonvilles:

[...] se alza un ruinoso tendido de informes viviendas, terrosas, obscuras, miserables. Con ligeras variantes, todas son iguales: sin ventanas, los muros roídos por el tiempo, de podridas maderas desajustadas formando anchas grietas por donde se cuela el frío y la lluvia, el viento y el polvo, el invierno y el desamparo (Belmar 1947: 37).

Le narrateur adopte un discours narratif fondé sur l'esthétique de la disparition et du vide: les énumérations, les asyndètes, les rythmes binaires et ternaires se multiplient. L'énonciation hachée, entrecoupée de virgules, agressée par de nombreuses occlusives ("podridas», "polvo», "desamparo») et des allitérations violentes («tendido», «terrosas», «informes», «formando», «frío») est à l'image de la crise qui ravage populations et habitations et dévore le village qui semble se réduire comme une peau de chagrin, anéanti par les circonstances économiques. La description de la maison du vendeur de chaussures, Vivanco, le confirme: «las vigas desnudas, ahumadas, muestran colgantes cendales de telarañas cuajadas de esqueletos de moscas [...]. Las hojas de periódicos y de revistas con que han empapelado los muros se ven rotas", "Aquí y allá, míseros muebles» (Belmar 1947: 64). Les lieux de vie s'effritent et se décomposent, tout comme leurs habitants. 


\section{Roble huacho, village anéanti}

À la misère, s'ajoutent les maladies. Les descriptions d'infirmités, d'affections ou d'autres blessures contribuent à créer une atmosphère de destruction. Les motifs narratifs de la misère, des maladies et de la laideur se combinent dans une description très zolienne. Ainsi, la population pauvre des faubourgs du village est-elle décrite comme: "una población horrenda, una sociedad de harapos y de hambre, piojosa, comida por la sarna y la miseria» (Belmar 1947: 37). La rage ou encore l'épilepsie affectent certains des personnages, par exemple le fils de la lavandière, Juan Manuel (Belmar 1947: 134) ou encore le fils de la villageoise Margarita, ausculté par Pancho dont le verdict est sans appel: «Es sarna, la vulgar, molesta sarna, que martiriza las noches de los pobres en el mundo» (Belmar 1947: 68). La dégradation est présentée comme un legs et les enfants de ces familles misérables sont décrits en ces termes: "la chiquillería gritona, siempre famélica», "casposas piernas de alambre y gruesas rodillas raquíticas» (Belmar 1947: 38). La maigreur et la faim népargnent ni les enfants ni leurs géniteurs et certaines des descriptions rappellent des tableaux du Siècle d'Or espagnol (Murillo, José de Ribera) et les "peintures noires» de Goya ${ }^{5}$. Le pharmacien Pancho est donc souvent sollicité par les villageois afin qu'il ausculte certains de leurs proches. En raison de la crise économique, le village est devenu un véritable désert médical où le pharmacien fait office de médecin: "Cuándo será el día en que se venga un médico a este pueblo desgraciado... Estoy transgrediendo la ley... No debo atender enfermos... Pero esta gente tampoco se puede morir así» (Belmar 1947: 66). Pancho, protagoniste et narrateur, se centre sur les descriptions médicales, lesquelles deviennent un procédé scriptural essentiel. La conséquence ultime de la crise est le décès, comme dans le cas du nourrisson dont Pancho raconte la venue au monde: "[...] y de pronto, como un vómito de sangre y de inmundicia, aparece una criatura sobre el tibio pellejo que lo espera» (Belmar 1947: 46). La monstruosité de la description semble ne laisser aucune issue aux populations touchées par la crise. Même la naissance est marquée par la destruction.

Les esthétiques de la laideur et de la décomposition deviennent des procédés scripturaux essentiels dans le roman. Les descriptions du narrateur, fortement expressives, intensifient les effets destructeurs de la crise. Avec une précision et une rigueur scientifiques, Pancho Ríos fait un portrait de la laideur, qui illustre bien l'analyse de R. Polin: "Ce qui est laid offusque, non pour ce qu'il n'a pas, mais pour ce qu'il a. Il est vraiment, non point absence de réalité, mais présence de laideur, non pas manque, mais trop plein» (Polin 1966: 260). Les descriptions de monstruosités et de corps difformes remplissent l'espace textuel: ainsi, le chef

5 Le traitement scriptural de la maladie est récurrent dans les années 1940 dans la littérature occidentale, comme dans La peste de Camus (1947). On retrouve également un intérêt pour l'écriture de la maladie physique et mentale mais surtout au XIX ${ }^{e}$ siècle dans la littérature française (Zola, Flaubert, Baudelaire) ainsi que dans l'Antiquité (Hérodote). 
de gare qui corrompt la jeune Armanda en lui proposant des rapports sexuels en échange de ses «hermosos billetes» se caractérise par ses: «manos horribles, feas cicatrices» (Belmar 1947: 44). Le vice semble donc se manifester également par le physique repoussant de cet homme. Quant à la fille du vendeur de chaussures, Vivanco, elle est atteinte de gonorrhée et Pancho décrit en détail le sexe infecté:

[...] un sexo en toda su cruda realidad, un sexo torcido, de pelos espesos y crespos; un sexo semejante a una boca deforma, deshumanizada, como la de algún horrible animal desconocido; una boca monstruosa, macerada, de labios negros; uno de ellos atrozmente hinchado, tumefacto, doloroso. De entre ellos fluye como un río lento y asqueroso, gruesa secreción lechosa y nauseabunda (Belmar 1947 : 65-66).

L'énumération, l'absence de verbes, l'abondance de virgules et les parallélismes de construction décomposent la syntaxe, reproduisant ainsi la déliquescence corporelle de la jeune fille. Cette esthétique de la déformation est perceptible aussi dans la description du fils de la lavandière Margarita: "deforme cabezota » (Belmar 1947: 67), "le ha aparecido una 'flota' de granos en todo el cuerpo", «repugnante», «rojas excoriaciones», «sarna» (Belmar 1947: 68). Tous les sens du lecteur sont sollicités pour partager avec le narrateur son dégoût mais aussi sa compassion envers les malheurs de cette population miséreuse. L'indien Cayupi, atteint de tuberculose, est décrit comme suit: «una tos seca, dolorosa, sacude a ratos, como una brizna, al indio mísero", "rostro consumido por la enfermedad, devorado por la fiebre» (Belmar 1947: 69). Enfin, le Guata Bascur apparait distordu dans une description grotesque: «descomunal desarrollo de su panza», "es un paquidermo lento y pesado, gigantesco, de patas tiesas por el reumatismo»(Belmar 1947: 70). La crise qui dégrade le monde semble se matérialiser et s'engouffrer dans les corps des individus, les décomposant à leur tour. Le langage est lui aussi déformé par cette même crise, comme le démontrent les nombreux chocs phoniques ( $k$, ch, rr) (Belmar 1947: 36), reflets du pourrissement qui atteint désormais l'espace de l'action: «Las rudas carretas fleteras de otro tiempo han desaparecido. Tal vez pudren sus maderas [...] al lado de las 'canchas' atiborradas de troncos derribados y cuarteados, hundidos, socavados [...], desmenuzados» (Belmar 1947: 36).

Tout le village est plongé dans la pénombre comme nous le constatons à plusieurs reprises, le mot "obscuridad» et ses dérivés revenant souvent: "la obscura silueta del convento franciscano", "el obscuro fondo de la noche" (Belmar 1947: 15, 19). L'espace géographique, mais aussi le silence et le souvenir sont marqués du sceau de l'obscurité, y compris les traits physiques de certains habitants, en particulier les yeux et la chevelure noirs. Michel Pastoureau rappelle que l'utilisation de la couleur est «culturelle et idéologique» et renvoie à des «enjeux économiques, politiques, sociaux, symboliques s'inscrivant dans un contexte précis» (Pastoureau 2008: 12).

Le texte semble s'auto-détruire par le biais de ces procédés discursifs qui font de la bourgade de Roble huacho un espace en pleine désagrégation. Roble huacho devient le village de tous les vices et des péchés capitaux. 
Paul Ricœur, qui étudie le concept de crise dans son acception large, l'analyse ainsi: "percevoir une situation comme crise, dirai-je dans la ligne de Landsberg et de Mounier, c'est ne plus savoir quelle est ma place dans l'univers, ne plus savoir quelle hiérarchie stable de valeurs peut guider mes préférences» (Ricœur 1988: 1-19). Dans le roman, effectivement, les valeurs sont en perdition et certains personnages en perte de repères en sont l'illustration. Lévocation de la luxure est par exemple récurrente. Les personnages se rendent dans les bas-fonds, à savoir la maison close tenue par Doña Eva et le contact des corps est suggéré par des allusions suffisamment explicites: «Siento junto a mi mejilla el contacto ardiente de un rostro febril y humedecido», "Aliro García [...] palmotea suavemente las gruesas nalgas que no dejan de temblar» (Belmar 1947: 22, 23). Sebastián Elgueta a une relation avec une mineure et s'en enorgueillit: «Estaba re buena... Se abrió como un lienzo » (Belmar 1947: 96-97). Cette référence érotique et l'obsession du désir charnel créent une deuxième crise au sein du roman: une crise familiale, car la tante de la Lucha, séduite par Elgueta, fait appel aux carabiniers afin qu'ils rétablissent l'ordre. Le paroxysme de la luxure ou de la perversion sexuelle est atteint lorsque «el rucio» Fernández raconte un cas de zoophilie: celui de Patas de Huasca copulant avec des poules (Belmar 1947: 31-32). En sus de la luxure et du péché capital, apparaissent divers exemples de vices comme le vol, lorsque Óscar Vegas, "tesorero comunal», puise dans les caisses du village (Belmar 1947: 52), ou l'alcoolisme, traditionnellement considéré tantôt comme vice, tantôt comme maladie et qui concerne bon nombre de personnages du roman: «el vino vuelve a llenar las copas» "Estoy borracho» (Belmar 1947: 56, 107). Ainsi, se produit-il un véritable bouleversement des valeurs, une crise morale qui découle tout naturellement de la crise économique, tout en étant en même temps un peu la cause de celle-ci, mais aussi de la Seconde Guerre mondiale, qui vient de prendre fin lors de la publication et donc non encore survenue dans l'histoire narrée. Le village est frappé par une malédiction, une sorte de châtiment divin comme le suggère le narrateur, lequel évoque les «dioses implacables» (Belmar 1947: 36) qui déterminent le destin tragique de Roble huacho. À travers la réécriture de la chute de Troie (dont les raisons économiques ont été transformées en raisons héroïques dans les poèmes de l'Antiquité), chute chantée dans l'Iliade, l'Odyssée et l'Enéide et dont les dieux sont en grande partie responsables, Roble huacho devient en quelque sorte, par l'implicite, un village mythifié, une sorte d'antichambre du Paradis, un Purgatoire, un lieu de passage obligé avant la survenue d'une époque meilleure.

\section{Récupérer une identité, rétablir la justice: résister face à la crise}

Pour mieux lutter contre la crise, les personnages réaffirment l'identité chilienne. Lanalyse d'Iván Carrasco peut permettre d'éclairer cette question de l'identité dans le roman de Belmar:

En el discurso literario estas identidades son representadas, configuradas o proyectadas principalmente en la actuación, caracterización y diálogo de personajes 
o tipos humanos representativos, en determinados enunciados ideológicos o valorativos de los sujetos enunciativos y en los discursos metatextuales complementarios del autor (Carrasco 2011:23-32).

C'est bien par le biais du langage que les protagonistes revendiquent leur identité. Il devient un instrument de cohésion sociale et une arme contre la décomposition de la société en proie à la crise. Les surnoms populaires, courants au Chili, donnés aux personnages reproduisent la familiarité conversationnelle: «el rucio» («blond»), «la Lucha», «Nacho Cabrera» (Belmar 1947: 145). Ce sont aussi des termes renvoyant aux traditions et à l'alimentation chiliennes: «el mote», boisson à base de grains de blé, «la once», sorte de goûter pratiqué au Chili, "la empanada», chausson à la viande, "la cazuela» et "el pebre», condiment, "la coliza», pain chilien, "los digüeñes», champignons, "los pequenes », petits chaussons à la viande (Belmar 1947: 40, 117, 85, 133, 106, 215). Les «chilenismes» (et parfois les termes d’origine mapuche) abondent: «está re buena", "al tiro», "esta cabra» (ibid., 102). Ils permettent aux personnages de se recentrer sur l'essentiel, de reconstruire un village voire un pays à partir de ce qui leur est le plus propre. La nourriture est lien social et, comme l'explique Barthes, "un véritable signe, c'est-à-dire peut-être l'unité fonctionnelle d'une structure de communication; [...] c'est toute la nourriture qui sert de signe entre les participants d'une population donnée» (Barthes 1961: 980). Le signe est, dans le cas qui nous intéresse, la conscience d'appartenir à une Histoire et à un pays, de partager une identité par l'alimentation (ce qui est valable aussi pour le langage), de s'unir en période de crise destructrice.

Le narrateur charpente également son discours narratif et l'espace textuel du roman en construisant une architecture du village : ainsi, évoque-t-il la «botica», la «plaza», l'«Hotel». Si le village est un lieu parfois infernal, les nombreuses descriptions d'un Paradis terrestre et d'un locus amoenus introduisent un havre de paix: «el paisaje se me entra por los ojos hasta el corazón», "el río es una vena abierta, palpitante, por donde fluye el agua viva y eterna " (Belmar 1947: 132-133), "un lujurioso oleaje de verdura sobre los valles», " una tierra convertida en flor» (Belmar 1947: 142). Ce débordement de vie et de couleurs surpasse la pénombre et la décomposition du village et de ses habitants. En outre, l'apparente structure fragmentaire de la narration se reconstruit à travers le rôle prépondérant de Pancho dans le roman. La riche galerie de personnages crée l'impression d'une explosion des sujets narratifs dont les expériences convergent cependant vers la figure de Pancho Ríos: le pharmacien-«médecin» est sollicité sans cesse par les villageois. Ses prescriptions médicales apaisent les «crises» de douleur de ses patients, mais il sait aussi, de façon symbolique, trouver des remèdes à une autre crise, la crise économique. Il redonne la santé à ceux qui lui rendent visite, c'est-à-dire à ceux qui sont les plus touchés par la dépression économique. Lincendie final de la pharmacie est d'autant plus symbolique: «Esto es el fin. Y el comienzo» (Belmar 1947: 221). Tel le Phénix, la pharmacie part en fumée, mais le feu purificateur et le cataclysme semblent annoncer une 
régénération pour le village désormais libéré de ses maux, et n’ayant plus besoin d’ordonnance médicale.

Pancho Ríos, enfin, se caractérise par son humanité et sa générosité, il donne de son temps aux villageois et refuse toute rémunération pour ses consultations (Belmar 1947: 67, 68). Il est un peu le fil qui maintient le village en vie et défend les pauvres gensqui le peuplent. Lorsqu'il est convoqué par la justice suite à l'inhumation du nouveau-né près d'une habitation, acte considéré comme illégal (Belmar 1947: 79, 83), il apparaît comme un nouveau Bon Samaritain: «Es una inhumación ilegal, por ignorancia. Me parece el colmo del rigor la detención de estas pobres mujeres por algo que no alcanza a ser un hecho delictuoso» (Belmar 1947 : 83). Pancho rétablit une justice, s'emploie à redonner la santé aux malades et affronte avec courage la crise et le monde sans pitié de la Justice.

\section{Conclusion}

Roble huacho apparaît comme un roman de résistance, une réaction face à la crise des années 1929-1933. Le titre du roman, qui est le nom du village où se déroule l'action, est symbolique:

En la misma esquina de la plaza, [...] un árbol aislado eleva hacia la altura su tronco centenario. El espeso y frondoso follaje es una copa de pájaros, un nidal de trinos y de alas, una guitarra verde y rumorosa en los dedos del viento.

Es un roble solitario, un roble 'huacho'. Ha entregado su nombre al pueblo que parece cobijarse bajo su sombra (Belmar 1947: 133).

Le chêne, unique arbre du village, est traditionnellement l'arbre de la justice. Inébranlable, il résiste aux assauts de la crise et de la misère, tout en conservant sa vitalité, comme le démontrent les termes «elevarse», "frondoso follaje», «trinos», «guitarra verde». Cet arbre solitaire, empreint de dynamisme, n’annoncerait-il pas une lueur d'espoir, le rétablissement d'une justice et la fin des souffrances des villageois?

Ce roman, reflet d'une époque marquée par la Grande Dépression et les deux guerres mondiales, restructure un univers dominé par le chaos. Le lyrisme de certaines évocations, l'histoire d'amour vécue par Pancho et la jeune Solveig, l'espace naturel où se réfugie le narrateur, son sens de la justice et sa générosité soulagent le discours narratif tourmenté. À travers la dénonciation des abus de la justice corrompue, représentée par le juge Walter (Belmar 1947: 75, 81), des grands propriétaires, représentés par Don Nacho Cabrera, le narrateur belmarien montre du doigt les méfaits du capitalisme et la corruption (Belmar 1947: 150, 154), la brutalité des oligarchies capables d'utiliser la violence pour faire taire les protestataires et leurs revendications. C'est le cas de Nacho Cabrera qui s'en prend au viejo Anguita: "La pesada argolla de la 'penca' cae arteramente sobre la frente venerable que se quiebra con un chasquido trágico» (Belmar 1947: 150). C'est que ce dernier a porté plainte contre le propriétaire au sujet de limites de terrains: «La demanda contra don 'Nacho' Cabrera fue acogida favorablemente 
en el juzgado de Temuco; el informe del agrimensor que vino a rectificar las líneas, influyó decisivamente en su favor pese a un intento de soborno por parte del rico» (Belmar 1947: 145). Cette brutalité verbale et physique trouve un écho dans la violence de la crise incarnée par ces hommes puissants qui en deviennent l'allégorie. Le roman "éclaire» donc bien «le concept de crise» dans son acception large: crise des valeurs, crise économique, crise narrative (fragmentation textuelle) et, à travers le prisme de son héros et narrateur, Pancho Ríos, Roble huacho démontre que la solidarité et l'altruisme sont un premier pas vers la résolution de toute crise.

\section{Bibliographie}

Barthes, R., 1961, «Pour une psycho-sociologie de l'alimentation contemporaine », Annales. Économies, Sociétés, Civilisations, n 5, p. 977-986.

Baudrillard, J., 1990, La Transparence du mal. Essai sur les phénomènes extrêmes, Paris, Galilée.

Belmar, D., 1947, Roble Huacho, Santiago du Chili, Cultura.

Bohoslavsky, E., 2002, "Desempleo, organización y política. Los trabajadores rurales del sur chileno frente a la Gran Depresión», Anuario de estudios americanos, Sevilla, vol. 59, no 2, p. 541-563.

Carrasco, I., «Identidades en la poesía canónica de Gabriela Mistral», Estudios filológicos, Valdivia, 48 (nov. 2011), p. 23-32.

Genette, G., 1982, Palimpsestes. La littérature au second degré, Éditions du Seuil, Points/ essais.

Louette, J.-F., 1995, Silences de Sartre, Presses Universitaires du Mirail, Essais de littérature, Cribles.

Morin, E., 1976, «Pour une crisologie», Communications, n 25, p. 149-163.

Orozco Jesús Vera M., "La narrativa de María Luisa Bombal: principales claves temáticas», Cauce, Revista de Filología y su Didáctica, n 12, 1989, p. 39-56.

Polin, R., 1966, «Rien n'est beau que le laid... ou presque », Revue d'esthétique, p. 258-265.

Pastoureau, M., 2008, Noir: histoire d'une couleur, Paris, Éditions du Seuil.

Ricœur, P., 1988, «La crise: un phénom Ricœur ène spécifiquement moderne?», Revue de théologie et de philosophie, 120, p. 1-19.

Riveros, L., 2009, «La gran depresión (1929-1932) en Chile», Facultad de Economía y negocios, Universidad de Chile, Departamento de Economía, Serie "Documentos de Trabajo", p. 1-25.

Sartre, J.-P., 1948, Qu'est-ce que la littérature?, Paris, Gallimard, Idées.

Sartre, J.-P., 1972, L'idiot de la famille, Paris, Gallimard.

Silva Castro, R., 1960, Evolución de las Letras chilenas. 1810-1960, Santiago, Editorial Andrés Bello. 
Sosnowski, S., 1997, Lectura crítica de la literatura americana. Vanguardias y toma de posesión, Caracas, Biblioteca Ayacucho.

Toso, R., Feller, Á., 1983, «La crisis económica de la década del treinta en Chile: lecciones de una experiencia», Santiago, Serie de Estudios Económicos, Documentos de investigación, $\mathrm{n}^{\circ}$ 21, p. 7-56.

Villalobos, S., 1983, Breve Historia de Chile, Santiago de Chile, Editorial Universitaria. 